\title{
Spacecraft Constrained Maneuver Planning Using Positively Invariant Constraint Admissible Sets
}

\author{
Avishai Weiss, Morgan Baldwin, R. Scott Erwin and Ilya Kolmanovsky
}

\begin{abstract}
The paper considers spacecraft motion planning based on the use of safe positively invariant sets. In this approach, a connectivity graph is constructed between a set of forced equilibria, forming a virtual net that is centered around a nominal orbital position. The connectivity between two equilibria is determined based on safe positively invariant sets in order to guarantee that transitions between equilibria can be effected while spacecraft actuator limits are adhered to and debris collisions are avoided. A graph search algorithm is implemented to find the shortest path around the debris. Simulation results are presented that illustrate this approach.
\end{abstract}

\section{INTRODUCTION}

With the growing amount of debris in Earth orbit, spacecraft maneuver planning procedures have to address debris avoidance requirements. While obstacle avoidance is a standard problem in robotics [2], [3], the related spacecraft problems have several unique features. In particular, the space environment is relatively uncluttered, thus permitting for a variety of maneuvers. Spacecraft dynamics are quite different from those of typical robots. Maneuver efficiency with respect to time and fuel consumption is a critical consideration. The states of the spacecraft and the debris can only be estimated, often with a significant estimation error. Finally, computational algorithms must be fast and optimized given moving objects and the limited computing power onboard most spacecraft. These unique features of spacecraft maneuver planning problems provide the motivation for the development of specialized algorithms.

In [1], we have introduced an on-board maneuver planning approach based on the use of constraint-admissible positively invariant sets to determine connectivity between a set of forced and unforced spacecraft equilibria forming a virtual net in the vicinity of the spacecraft. Two equilibria are connected if a choice of a Linear Quadratic (LQ) feedback gain can be made that results in a transition between the equilibria which avoids the debris collision and satisfies the limits on thrust. The connectivity graph for all the equilibria in the net is constructed and real-time graph search algorithms are used to optimize an equilibria hopping sequence that avoids the debris collisions.

Unlike the open-loop trajectory optimization approaches, we do not rely on precise assignment of spacecraft position

A. Weiss is a Graduate Student, Department of Aerospace Engineering, The University of Michigan, Ann Arbor, MI.

M. Baldwin is a Research Aerospace Engineer, Space Vehicles Directorate, Air Force Research Laboratory, Albuquerque, NM.

R. Scott Erwin is a Principal Research Aerospace Engineer, Space Vehicles Directorate, Air Force Research Laboratory, Albuquerque, NM.

I. Kolmanovsky is a Professor, Department of Aerospace Engineering, The University of Michigan, Ann Arbor, MI. to the time instants along the trajectory, but instead switch to the next set-point and controller gain once appropriate conditions are satisfied. While this approach is conservative, it facilitates fault-tolerant and disturbance-tolerant execution of the maneuvers. Furthermore, by using disturbance-invariant sets [13] in the construction, we can assure robustness to unmeasured (but set-bounded) disturbances and uncertainties. This extension to handling unmeasured disturbances and uncertainties using techniques of [13] is not pursued here and is left to a longer version of this paper.

To facilitate the on-board computations of the connectivity graph, a fast growth distance computation procedure between two ellipsoidal sets has been proposed in [1]. In this approach, using the Karush-Kuhn-Tucker conditions, the growth distance computations are reduced to a root finding problem for the scalar value of the Lagrange multiplier. Then a predictor-corrector dynamic Newton-Raphson algorithm is used to update the Lagrange multiplier thereby rapidly estimating the growth distance from different equilibria in the virtual net to the debris.

In this paper, we incorporate limited thrust requirements into the computation of thrust limit on the growth distance, and we simulate maneuvers that adhere to the limited thrust constraints. Even though the computation of thrust limits on the growth distance can be performed offline for the nominal operating conditions, fast computational procedures are beneficial in case of thruster failures, degradations, and restrictions on thrust directions (e.g., caused by the presence of other spacecraft nearby), all of which can lead to changing constraints on thrust during spacecraft missions. If the thrust limits are prescribed in the form of 2-norm bounds, the optimization problem involved in computing the thrust limit on the growth distance is non-convex (unlike computing the growth distance itself). Consequently, we advocate the use of polyhedral norm bounds on thrust, that lend themselves to explicit solutions.

The related literature on spacecraft trajectory optimization with obstacle/debris avoidance is surveyed in [1]. Previous research addresses topics in spacecraft trajectory optimization [5], collision avoidance strategies based on risk assessment [6], the use of artificial potential functions [7], [8], and the use of conventional and mixed integer linear programming techniques [9], [10], [11], [12].

The paper is organized as follows. In Section II we discuss the nonlinear and the linearized models used to represent spacecraft relative motion dynamics. In Section III we review our approach to constructing the virtual net based on a set of forced and unforced equilibria and using this virtual net 
for debris avoidance. The procedure to compute the thrust limit on the growth distance is presented. Simulation results are reported in Section IV. Finally, concluding remarks are made in Section V.

\section{Relative Motion Model}

The spacecraft relative motion model represents the spacecraft dynamics in the (non-inertial) Hill's frame with the origin at a target location on a nominal circular orbit. For small distances, the linearization of the relative motion model given by the Hill-Clohessy-Wiltshire (CWH) equations is used [14].

\section{A. Nonlinear equations of motion}

The relative position vector of the spacecraft with respect to a target location on a circular orbit is expressed as

$$
\delta \vec{r}=x \hat{\imath}+y \hat{\imath}+z \hat{k},
$$

where $x, y$ and $z$ are the components of the position vector of the spacecraft relative to the target location and $\hat{\imath}, \hat{\jmath}, \hat{k}$ are the unit vectors of the Hill's frame. The Hill's frame has its $x$-axis along the orbital radius, $y$-axis along the orbital track, and $z$-axis is orthogonal to orbital plane.

The position vector of the spacecraft with respect to the center of the Earth can be expressed as $\vec{R}=\vec{R}_{0}+\delta \vec{r}=$ $\left(R_{0}+x\right) \hat{\imath}+y \hat{\jmath}+z \hat{k}$, where $R_{0}$ is the nominal orbital radius. The nonlinear equations of motion for the spacecraft (relative to an inertial frame) can be expressed in vector form as

$$
\ddot{\vec{R}}=-\mu \frac{\vec{R}}{R^{3}}+\frac{1}{m_{c}} \vec{F},
$$

where $\vec{F}$ is the vector of external forces applied to the spacecraft, $R=|\vec{R}|, m_{c}$ is the mass of the spacecraft, $\mu$ is the gravitational constant, and

$$
\ddot{\vec{R}}=\left(\ddot{x}-2 n \dot{y}-3 n^{2} x\right) \hat{\imath}+(\ddot{y}+2 n \dot{x}) \hat{\jmath}+\left(\ddot{z}+n^{2} z\right) \hat{k} .
$$

In these equations, $n=\sqrt{\frac{\mu}{R_{0}^{3}}}$ denotes the mean motion of the nominal orbit. Similar equations can be used to describe the motion of the debris.

\section{B. Linearized HCW equations in discrete-time}

For $\delta r \quad<R$, the linearized Hill-Clohessy-Wiltshire (HCW) equations [14] approximate the relative motion of the spacecraft on a circular orbit as

$$
\begin{gathered}
\ddot{x}-3 n^{2} x-2 n \dot{y}=\frac{F_{x}}{m_{c}}, \\
\ddot{y}+2 n \dot{x}=\frac{F_{y}}{m_{c}}, \\
\ddot{z}+n^{2} z=\frac{F_{z}}{m_{c}},
\end{gathered}
$$

where $F_{x}, F_{y}, F_{z}$ are components of the external force vector (excluding gravity) acting on the spacecraft. The linearized dynamics account for differences in gravity between the spacecraft and nominal orbital location, and for relative motion effects. The spacecraft relative motion dynamics in the orbital plane $(x$ and $y$ ) and in the out-of-orbital plane (z) are decoupled. The in-plane dynamics are Lyapunov unstable, while the out-of-plane dynamics are Lyapunov stable. The in-plane dynamics are completely controllable from $F_{y}$ input but are not controllable from $F_{x}$ input. The out-of-plane dynamics are controllable from $F_{z}$ input. These dynamics are thus different from typical robots.

Assuming a sampling period of $\Delta T$ sec, we can convert the model (2) to a discrete-time form

$$
X(t+1)=A X(t)+B U(t)
$$

where $X(t)=[x(t), \quad y(t), \quad z(t), \quad \dot{x}(t), \quad \dot{y}(t), \dot{z}(t)]^{\mathrm{T}}$ is the state vector at the time instant $t \in Z^{+}, U(t)=$ $\left[F_{x}(t), F_{y}(t), F_{z}(t)\right]^{\mathrm{T}}$ is the control vector of thrust forces at the time instant $t \in Z^{+}$, and $A=\exp \left(A_{c} \Delta T\right), B=$ $\int_{0}^{\Delta T} \exp \left(A_{c}(\Delta T-\tau)\right) d \tau B_{c}$ are the discretized matrices obtained based on the continuous-time system realization $\left(A_{c}, B_{c}\right)$. Alternatively, the control vector $U$ can represent an instantaneous change in the velocity of the spacecraft, $\Delta v$, induced by thrust, with an appropriately re-defined $B$-matrix,

$$
B_{\Delta v}=e^{A_{c} \Delta T}\left[\begin{array}{lll}
0 & 0 & 0 \\
0 & 0 & 0 \\
0 & 0 & 0 \\
1 & 0 & 0 \\
0 & 1 & 0 \\
0 & 0 & 1
\end{array}\right]
$$

\section{DEBRis AVOIDANCE BASED ON A ViRtuAl Net}

Our approach to debris avoidance is based on utilizing constraint-admissible positively invariant sets [13], [15] centered around the spacecraft forced and unforced equilibria. A finite set of these equilibria used for constructing debris avoidance maneuvers is referred to as a virtual net. Given an estimate of the debris position, we build a connectivity graph that identifies the equilibria in the virtual net between which the spacecraft can move, with guaranteed collisionfree motion and within the available thrust authority. We then employ graph search to determine an efficient path between the equilibria that ensures debris avoidance.

\section{A. Virtual Net}

The virtual net comprises a finite set of equilibria, $X_{e}(r)$, corresponding to a finite set of prescribed spacecraft positions $r \in \mathcal{N}=\left\{r_{1}, r_{2}, \ldots, r_{n}\right\} \subset R^{3}$,

$$
X_{e}\left(r_{k}\right)=\left[\begin{array}{c}
r_{k} \\
0
\end{array}\right]=\left[\begin{array}{c}
r_{k x} \\
r_{k y} \\
r_{k z} \\
0 \\
0 \\
0
\end{array}\right], k=1, \cdots, n
$$

whose velocity states are zero, and where $n$ is the number of equilibria in the virtual net. See Figure 1. We assume that for all $r \in \mathcal{N}$, the corresponding values of control necessary to support the specified equilibria in steady-state satisfy the imposed thrust limits. 


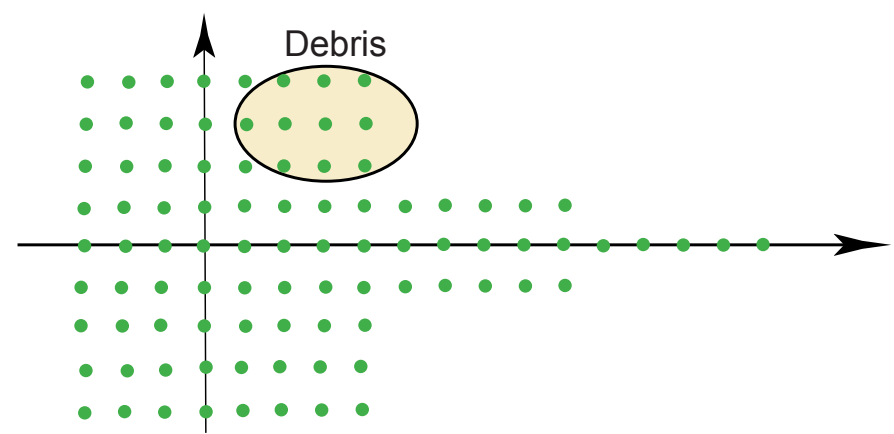

Fig. 1: The virtual net for debris avoidance. Dots correspond to positions at equilibria, $X_{e}(r)$, on a virtual net. The ellipsoid represents the debris position and uncertainty.

\section{B. LQ Controller with Gain Switching}

A conventional Linear-Quadratic (LQ) feedback is used to control the spacecraft to a commanded equilibrium in (4),

$$
U=K\left(X-X_{e}(r)\right)+\Gamma r=K X+H(K) r,
$$

where

$$
\begin{gathered}
\Gamma=\left[\begin{array}{ccc}
-3 n^{2} m_{c} & 0 & 0 \\
0 & 0 & 0 \\
0 & 0 & n^{2} m_{c}
\end{array}\right], \\
H(K)=\Gamma-K\left[\begin{array}{c}
I_{3} \\
0_{3}
\end{array}\right],
\end{gathered}
$$

and where $I_{3}$ denotes the $3 \times 3$ identity matrix and $0_{3}$ denotes the $3 \times 3$ zero matrix. This LQ controller provides an asymptotically stable closed-loop system but does not enforce the constraints.

To provide greater flexibility in handling constraints, a multimode controller architecture is employed [15]. Specifically, we assume that a finite set of LQ gains $K \in \mathcal{K}=$ $\left\{K_{1}, \cdots, K_{m}\right\}$ is available to control the spacecraft. By using a large control weight in the LQ cost functional, motions with low fuel consumption yet large excursions can be generated; using a large control weight in the LQ cost, motions with short transition time can be generated [16]. We assume that a preference ordering has been defined and the gains are arranged in the order of descending preference, from $K_{1}$ being the highest preference gain to $K_{m}$ being the lowest preference gain.

\section{Positively Invariant Sets}

The ellipsoidal set

$$
\begin{aligned}
& \bar{C}(r, K)= \\
& \left\{X \in R^{6}: \frac{1}{2}\left(X-X_{e}(r)\right)^{T} P(K)\left(X-X_{e}(r)\right) \leq 1\right\} \subset R^{6},
\end{aligned}
$$

where $(A+B K)^{T} P(A+B K)-P<0, P=P(K)>0$, is positively invariant. Positive invariance implies that any trajectory of the closed-loop system that starts in $\bar{C}(r, K)$ is guaranteed to stay in $\bar{C}(r, K)$ as long as the same LQ gain $K$ is used and the set-point command $r$ is maintained. To achieve the positive invariance, the matrix $P$ can be obtained as the solution of the discrete-time Riccati equation or of the above Lyapunov equation for the closed-loop asymptotically stable system. We note that, because the system is linear, the positive invariance of $\bar{C}(r, K)$ implies the positive invariance of the scaled set

$$
\begin{gathered}
C(r, K, \rho)=\left\{X \in R^{6}: \frac{1}{2}\left(X-X_{e}(r)\right)^{T} P(K)\left(X-X_{e}(r)\right)\right. \\
\left.\leq \rho^{2}\right\}, \quad \rho \geq 0 .
\end{gathered}
$$

Geometrically, the set $C(r, K, \rho)$ corresponds to an ellipsoid scaled by the value of $\rho$ and centered around $X_{e}(r), r \in \mathcal{N}$.

\section{Debris Representation}

We use a set, $O(z, Q)$, centered around the position $z \in$ $R^{3}$, to over-bound the position of the debris, i.e.,

$$
O(z, Q)=\left\{X \in R^{6}:(S X-z)^{T} Q(S X-z) \leq 1\right\},
$$

where $Q=Q^{\mathrm{T}}>0$ and

$$
S=\left[\begin{array}{llllll}
1 & 0 & 0 & 0 & 0 & 0 \\
0 & 1 & 0 & 0 & 0 & 0 \\
0 & 0 & 1 & 0 & 0 & 0
\end{array}\right]
$$

The set $O(z, Q)$ can account for the debris and spacecraft physical sizes and also for the uncertainties in the estimation of the debris/spacecraft position. Note that the set $O(z, Q)$ has an ellipsoidal shape in the position directions and it has a cylindrical shape in the velocity directions. Ellipsoidal sets rather than polyhedral sets are used to over-bound the debris since ellipsoidal bounds are typically produced by position estimation algorithms, such as the Extended Kalman Filter (EKF).

\section{E. Debris Avoidance Approach}

Consider now $r_{i} \in \mathcal{N}$, representing a possible position on the net that the spacecraft can move to as a part of the debris avoidance maneuver. Suppose that the current state of the spacecraft is $X\left(t_{0}\right)$ at the time instant $t_{0} \in Z^{+}$. If there exists a $\rho \geq 0$ and $K_{j} \in \mathcal{K}$ such that

$$
X\left(t_{0}\right) \in C\left(r_{i}, K_{j}, \rho\right) \text { and } O(z, Q) \cap C\left(r_{i}, K_{j}, \rho\right)=\emptyset,
$$

the spacecraft can move to the position $r_{i} \in \mathcal{N}$ by engaging the control law with $r(t)=r_{i}$ and $K(t)=K_{j}, t \geq t_{0}$, and without hitting the debris confined to $O(z, Q)$. This idea underlies our subsequent approach to debris avoidance, where we maintain the spacecraft trajectories within the tube formed by positively invariant sets that do not overlap with the debris.

To avoid a non-stationary debris, its path can be covered by a union of a finite number of sets of ellipsoidal shape,

$$
\mathcal{D}=\bigcup_{l=1}^{l=n_{d}} O\left(z_{l}, Q_{l}\right)
$$

where the center of the $l$ th set is denoted by $z_{l} \in R^{3}$ and the lth set shape is defined by $Q_{l}=Q_{l}^{\mathrm{T}}>0$. Then the debris 
path avoidance condition is

$$
\begin{gathered}
X\left(t_{0}\right) \in C\left(r_{i}, K_{j}, \rho\right) \text { and } O\left(z_{l}, Q_{l}\right) \cap C\left(r_{i}, K_{j}, \rho\right)=\emptyset \\
\quad \text { for all } l=1, \cdots, n_{d}
\end{gathered}
$$

The same approach, with larger $n_{d}$, can be used to handle multiple non-stationary debris. Note, however, that this approach is conservative as it does not account for the debris progression along its path.

\section{F. Growth Distances}

The minimum value of $\rho \geq 0$ for which $O(z, Q) \bigcap C(r, K, \rho) \neq \emptyset$ is referred to as the growth distance, similarly to [17]. This growth distance can also be viewed as the least upper bound on the values of $\rho$ for which $O(z, Q)$ and $C(r, K, \rho)$ do not intersect. See Figure 2. We use the notation $\rho_{g}(r, K, Q, z)$ to reflect the dependence of the growth distance on the set-point $r \in \mathcal{N}$, the control gain $K \in \mathcal{K}$ and the obstacle parameters $Q$ and $z$.

Note that the growth distance depends on the position of the debris which may be unknown in advance. Consequently, the growth distance computations have to be performed online. The computations of the growth distance, $\rho_{g}(r, K, Q, z)$, have been addressed in [1]; for completeness, these computations are summarized in this paper.

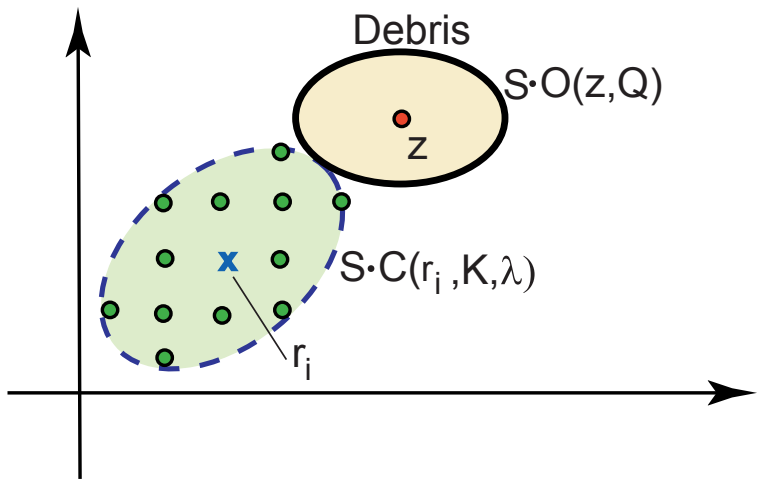

Fig. 2: The positively invariant set is grown till touching the debris. The spacecraft can move from any of the equilibria on the virtual net inside the positively invariant set $C(r, K, \rho)$ to $X_{e}\left(r_{i}\right)$ marked by 'x' without colliding with the debris.

Since the spacecraft maneuvers have to be performed using limited thrust, we additionally define a maximum value of $\rho=\rho_{u}(r, K)$ for which $X \in C\left(r, K, \rho_{u}(r, K)\right)$ implies that the thrust $U=K X+H(K) r$ satisfies the imposed thrust limits. We refer to $\rho_{u}$ as the thrust limit on growth distance. Unlike $\rho_{g}$, the value of $\rho_{u}$ does not depend on the position or shape of the debris and can be pre-computed off-line.

Finally, we define the thrust limited growth distance

$$
\rho^{*}(r, K, Q, z)=\min \left\{\rho_{g}(r, K, Q, z), \rho_{u}(r, K)\right\} .
$$

Note that $X\left(t_{0}\right) \in C\left(r_{i}, K_{j}, \rho^{*}\left(r_{i}, K_{j}, z\right)\right)$ implies that the ensuing closed-loop spacecraft trajectory under the control (5) with $r(t)=r_{i}$ and $K(t)=K_{j}$ for $t \geq t_{0}$ satisfies the thrust limits and avoids debris collisions for a debris confined to $O(z, Q)$.

The above definitions were given for the case of a single stationary debris, $O(z, Q)$. In the case of multiple debris or when avoiding a predicted debris path based on (10), the growth distance is replaced by the multi-growth distance, which is the minimum growth distance to each of $O\left(z_{l}, Q_{l}\right)$, $l=1, \cdots, n_{d}$.

\section{G. Growth Distance Computations}

Define $\bar{X}=X-X_{e}(r)$ and $\alpha=2 \rho^{2}$. The problem of determining the growth distance $\rho_{g}(r, K, Q, z)$, reduces to the following constrained optimization problem:

$$
\begin{gathered}
\min _{\alpha, \bar{X}} \alpha \\
\text { subject to } \\
\bar{X}^{\mathrm{T}} P \bar{X} \leq \alpha, \\
\left(( S ( \overline { X } + X _ { e } ( r ) ) - z ) ^ { \mathrm { T } } Q \left(\left(S\left(\bar{X}+X_{e}(r)\right)-z\right) \leq 1 .\right.\right.
\end{gathered}
$$

To solve this optimization problem, we use the KarushKuhn-Tucker (KKT) conditions. Note that the standard linear independence constraint qualification conditions hold given that $P>0$. We define

$$
\begin{aligned}
& \mathcal{L}=\alpha+\lambda_{1}\left(\bar{X}^{\mathrm{T}} P \bar{X}-\alpha\right) \\
& +\lambda_{2}\left(\left(S\left(\bar{X}+X_{e}(r)\right)-z\right)^{\mathrm{T}} Q\left(S\left(\bar{X}+X_{e}(r)\right)-z\right)-1\right)
\end{aligned}
$$

where $\lambda_{1}$ and $\lambda_{2}$ are Lagrange multipliers. The stationarity of the Lagrangian (setting partial derivative equal to zero) with respect to $\alpha$ yields $\lambda_{1}=1$. The stationarity of the Lagrangian with respect to $\bar{X}$ leads to [1]:

$\bar{X}=\bar{X}\left(\lambda_{2}, r, z\right)=-\left(P+\lambda_{2} S^{\mathrm{T}} Q S\right)^{-1} S^{\mathrm{T}} Q\left(S X_{e}(r)-z\right) \lambda_{2}$,

where $\lambda_{2} \geq 0$ is a scalar to be determined. Note that $P>0$, $S^{\mathrm{T}} Q S \geq 0, \lambda_{2} \geq 0$ (as the Lagrange multiplier corresponding to an inequality constraint) imply that $\left(P+\lambda_{2} S^{\mathrm{T}} Q S\right)$ is invertible. The problem reduces to finding a nonnegative scalar $\lambda_{2}$ which is the root of the equation

$$
F\left(\lambda_{2}, r, z\right)=\left((S X-z)^{\mathrm{T}} Q(S X-z)-1=0,\right.
$$

where

$$
X=\bar{X}\left(\lambda_{2}, r, z\right)+X_{e}(r)
$$

The scalar root finding problem (15) has to be solved online multiple times for different $r \in \mathcal{N}$, and in the case of avoiding a predicted debris path also for different $z$ 's. To solve this problem fast and to re-use the previously found solutions as approximations, in [1] we have proposed a dynamic Newton-Raphson's algorithm. This algorithm uses predictor-corrector updates to track the root as a function of 
$z$ and $r$ :

$$
\begin{aligned}
\lambda_{2}^{k+1,+} & =\lambda_{2}^{k}+\left\{\frac{\partial F}{\partial \lambda_{2}}\left(\lambda_{2}^{k}, z^{k}, r^{k}\right)\right\}^{-1}\left\{-F\left(\lambda_{2}^{k}, z^{k}, r^{k}\right)\right. \\
& -\frac{\partial F}{\partial z}\left(\lambda_{2}^{k}, z^{k}, r^{k}\right)\left(z^{k+1}-z^{k}\right) \\
& \left.-\frac{\partial F}{\partial r}\left(\lambda_{2}^{k}, z^{k}, r^{k}\right)\left(r^{k+1}-r^{k}\right)\right\}, \\
\lambda_{2}^{k+1} & =\max \left\{0, \lambda_{2}^{k+1,+}\right\} .
\end{aligned}
$$

To implement the algorithm, we take advantage of known functional form for $F$ and compute explicitly the partial derivatives,

$$
\begin{gathered}
\frac{\partial \bar{X}}{\partial \lambda_{2}}=\left(P+\lambda_{2} S^{\mathrm{T}} Q S\right)^{-1}\left\{-S^{\mathrm{T}} Q\left(S X_{e}(r)-z\right)\right\}, \\
\frac{\partial F}{\partial \lambda_{2}}=2(S \bar{X}-r)^{\mathrm{T}} Q\left(S \frac{\partial \bar{X}}{\partial \lambda_{2}}\right), \\
\frac{\partial \bar{X}}{\partial r}=\left(P+\lambda_{2} S^{\mathrm{T}} Q S\right)^{-1}\left\{-S^{\mathrm{T}} Q S \Omega\right\} \lambda_{2}, \\
\frac{\partial F}{\partial r}=2(S \bar{X}-z+r)^{\mathrm{T}} Q\left(S \frac{\partial \bar{X}}{\partial r}+I_{3}\right), \\
\frac{\partial \bar{X}}{\partial z}=\left(P+\lambda_{2} S^{\mathrm{T}} Q S\right)^{-1} S^{\mathrm{T}} Q S \Omega \lambda_{2}, \\
\frac{\partial F}{\partial z}=2(S \bar{X}-z+r)^{\mathrm{T}} Q\left(S \frac{\partial \bar{X}}{\partial z}-I_{3}\right) .
\end{gathered}
$$

In these equations,

$$
X_{e}(r)=\Omega r, \text { where } \Omega=\left[\begin{array}{c}
I_{3} \\
0
\end{array}\right],
$$

and $I_{3}$ denotes the $3 \times 3$ identity matrix.

Figures 4-5 illustrate the growth distance tracking in the case when $z=[0,0,0]^{\mathrm{T}}, Q=4 I_{3}$, and $P=P^{\mathrm{T}}>0$ is determined as a solution to the Lyapunov equation,

$$
\begin{aligned}
& P= \\
& \left(\begin{array}{cccccc}
530.67 & 0.066 & 0 & -14.97 & -0.69 & 0 \\
0.066 & 531.17 & 0 & 0.69 & -15.03 & 0 \\
0 & 0 & 531.04 & 0 & 0 & -15.06 \\
-14.97 & 0.69 & 0 & 1.43 & 0 & 0 \\
-0.69 & -15.03 & 0 & 0 & 1.43 & 0 \\
0 & 0 & -15.06 & 0 & 0 & 1.43
\end{array}\right) .
\end{aligned}
$$

For the first 20 iterations, $r^{k}$ is a constant to enable initial convergence of the algorithm to take place. Then $r^{k}$ starts to vary through the virtual net, see Figure 3 . One iteration of the Newton-Raphson algorithm per value of $r^{k}$ is used to update the root, $\lambda_{2}^{k+1}$. Figure 4 demonstrates that the growth distance tracking is accurate. The growth distance occasionally becomes zero indicating the overlap between the debris and several of $r^{k}$. This is confirmed from Figure 5, which illustrates the trajectory of $r^{k}$ in the three dimensional Hills' frame relative to the debris.

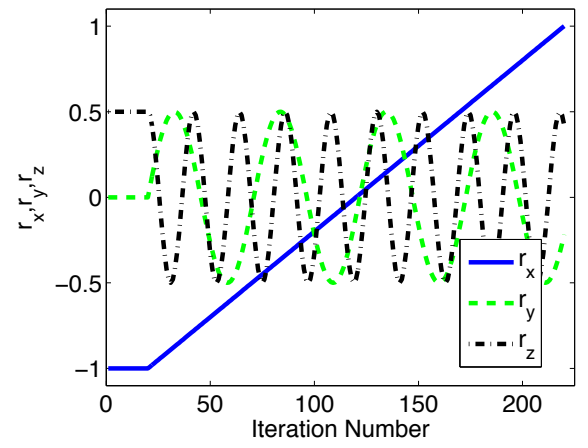

Fig. 3: Components of $r, r_{x}, r_{y}$ and $r_{z}$ varying versus the iteration number.

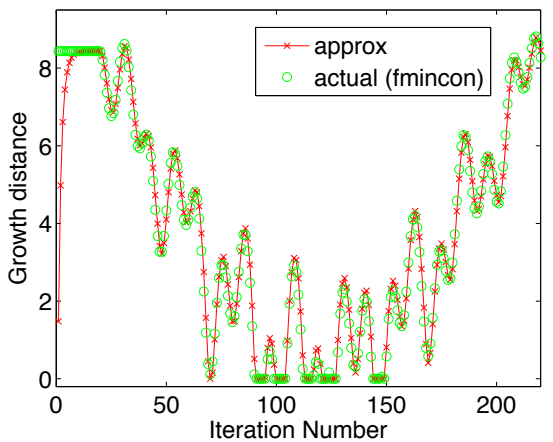

Fig. 4: Growth distance versus iteration number computed by dynamic Newton-Raphson algorithm.

\section{H. Thrust Limit on Growth Distance Computations}

In [1], the following procedure to compute $\rho_{u}(r, K)$ has been discussed. Suppose that the thrust limits are expressed in the form $\|L U\| \leq 1$ for an appropriately defined matrix $L$ and norm $\|\cdot\|$. The computational procedures to determine $\rho_{u}(r, K)$ involves solving a bilevel optimization problem where $\|L(K X+H(K) r)\|$ is maximized subject to the constraint $X \in C(r, K, \alpha)$ and bisections are performed on the value of $\alpha$ so that the maximum value is driven to 1. As we demonstrate in this paper, in special cases this computation can be greatly simplified.

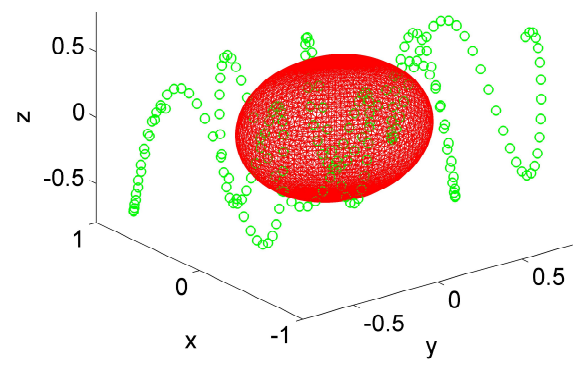

Fig. 5: The trajectory of $r$ and the debris. 
Suppose that the thrust constraints are prescribed in terms of polyhedral norm bounds, specifically, as

$$
e_{i}^{\mathrm{T}}(K X+H r) \leq u_{\max }, i=1,2, \cdots, m,
$$

where $e_{i}$ are the vertices of the unit norm polytope and $u_{\max }$ is the norm bound. The infinity norm, for instance, has $m=$ 6 , and

$$
\begin{aligned}
& e_{1}=\left[\begin{array}{l}
1 \\
0 \\
0
\end{array}\right] \quad e_{2}=\left[\begin{array}{c}
-1 \\
0 \\
0
\end{array}\right] \quad e_{3}=\left[\begin{array}{l}
0 \\
1 \\
0
\end{array}\right] \\
& e_{4}=\left[\begin{array}{c}
0 \\
-1 \\
0
\end{array}\right] \quad e_{5}=\left[\begin{array}{l}
0 \\
0 \\
1
\end{array}\right] \quad e_{6}=\left[\begin{array}{c}
0 \\
0 \\
-1
\end{array}\right] \text {. }
\end{aligned}
$$

In the case of non-polyhedral norm bounds, such as the 2norm, an approximation by a polyhedral norm bound can be employed.

Then, the determination of the thrust limit on the growth distance is based on solving a sequence of the following optimization problems for $i=1, \cdots, n$,

$$
e_{i}^{\mathrm{T}}(K X+H r) \rightarrow \max ,
$$

subject to

$$
\frac{1}{2}\left(X-X_{e}(r)\right)^{\mathrm{T}} P\left(X-X_{e}(r)\right) \leq c .
$$

If the value of $c$ is found for which the solutions of (19)(20), $X_{i}^{*}$, satisfy $\max _{i}\left\{e_{i}^{\mathrm{T}}\left(K X_{i}^{*}+H r\right)\right\}=u_{\max }$, then $\rho_{u}(r, K)=\sqrt{c}$. The problem (19)-(20) can be solved by diagonalizing $P$, using an orthogonal matrix, $V$,

$$
P=V^{\mathrm{T}} \Lambda V, \Lambda=\operatorname{diag}\left[\lambda_{1}^{2}, \cdots, \lambda_{6}^{2}\right], \lambda_{i}>0 .
$$

By defining, $z=X-X_{e}(r)$, and $\zeta$ so that

$$
z=V^{\mathrm{T}} \Lambda^{-\frac{1}{2}} \zeta
$$

it follows that

$$
\begin{aligned}
z^{\mathrm{T}} P z & =\zeta^{\mathrm{T}} \Lambda^{-\frac{1}{2}} V P V^{\mathrm{T}} \Lambda^{-\frac{1}{2}} \zeta \\
& =\zeta^{\mathrm{T}} \zeta .
\end{aligned}
$$

The problem (19)-(20) can now be re-written as

$$
h_{i}^{\mathrm{T}} \zeta+e_{i}^{\mathrm{T}} \Gamma r \rightarrow \max ,
$$

subject to

$$
\frac{1}{2} \zeta^{\mathrm{T}} \zeta \leq c
$$

where

$$
h_{i}^{\mathrm{T}}=e_{i}^{\mathrm{T}} K V^{\mathrm{T}} \Lambda^{-\frac{1}{2}} .
$$

The solution to the constrained maximization problem (21)-(22) of maximizing the inner-product of two vectors over a unit 2-norm ball is given by

$$
\zeta_{i}=\frac{h_{i}}{\left\|h_{i}\right\|} \sqrt{2 c},
$$

where $\|\cdot\|$ denotes the vector 2 -norm. The maximum value of the objective function is

$$
\left\|h_{i}\right\| \sqrt{2 c}+e_{i}^{\mathrm{T}} \Gamma r .
$$

Consequently, to satisfy (17), we use the following relations:

$$
\begin{gathered}
c=0 \text { if for any } i, u_{\max } \leq e_{i}^{\mathrm{T}} \Gamma r . \\
c=\min _{i} \frac{1}{2}\left(\frac{u_{\max }-e_{i}^{\mathrm{T}} \Gamma r}{\left\|h_{i}\right\|}\right)^{2} \text { otherwise. }
\end{gathered}
$$

Thus the problem of finding the thrust limit on the growth distance, when polyhedral norm bounds on thrust are employed, has an explicit solution given by (24).

We note that the condition $u_{\max } \geq \max _{i}\left\{e_{i}^{\mathrm{T}} \Gamma r\right\}$ is satisfied if the available thrust can maintain the equilibrium $X_{e}(r)$ in steady-state. We also note that, based on the form of $\Gamma, c$ is independent of $r_{y}$, the in-track component of the equilibrium in the virtual net. Hence the computations of $\rho_{u}(r, K)$ need to be only performed with $r_{y}=0$.

In the case when the spacecraft does not have independent thrusters in $x, y$ and $z$ directions, the 2-norm thrust limit is more practical. Unfortunately, maximizing a quadratic function in (19) subject to (20) is, in general, a non-convex problem. In this case, the 2-norm bound can be approximated by a polyhedral norm bound (17), with the vertices $e_{i}$ selected on the unit 2-norm ball in $R^{3}$. We note that higher accuracy of this approximation requires a higher number of vertices in (17), which thus, complicates (24).

Finally, we note that in the case the $\Delta v$ 's are treated as control inputs, the thrust limit on the growth distance is induced by the available $\Delta v$. Computing the thrust limit on the growth distance in this case is completely analogous to computing it in the case when the control input is the thrust force or thrust acceleration.

\section{Connectivity Graph and Graph Search}

We now introduce a notion of connectivity between two vertices of the virtual net, $r_{i} \in \mathcal{N}$ and $r_{j} \in \mathcal{N}$. The vertex $r_{i}$ is connected to the vertex $r_{j}$ if there exists a gain $K \in \mathcal{K}$ such that

$$
X_{e}\left(r_{i}\right) \in \operatorname{int} C\left(r_{j}, K, \rho^{*}\left(r_{j}, K, z\right)\right),
$$

where int denotes the interior of a set. The connectivity implies that a spacecraft located close to an equilibrium corresponding to $r_{i}$ can transition to an equilibrium $X_{e}\left(r_{j}\right)$ by using limited thrust and avoiding collision with the debris. We note that if $r_{i}$ is connected to $r_{j}$ this does not imply that, in turn, $r_{j}$ is connected to $r_{i}$. We also note that connectivity depends on the existence of an appropriate control gain from the set of gains $\mathcal{K}$ but the condition (25) does not need to hold for all gains.

The on-line motion planning with debris avoidance is performed according to the following procedure (for simplicity, described here for the case of a single debris):

Step 1: Determine the debris location and shape (i.e., $z$ and $Q$ ).

Step 2: By using fast growth distance computations, determine thrust limited growth distance based on (12), with $\rho_{g}$ computed online and $\rho_{u}$ precomputed off-line.

Step 3: Construct a graph connectivity matrix between all $r_{i}, r_{j} \in \mathcal{N}$. In the graph connectivity matrix, if 
two vertices are not connected, the corresponding matrix element is zero; if they are connected the corresponding matrix element is 1 . In parallel, build the control gain selectivity matrix, which identifies the index of the highest preference gain $K$ for which $r_{i}$ and $r_{j}$ are connected. This gain will be applied if the edge connecting $r_{i}$ and $r_{j}$ is traversed.

Step 4: Perform graph search to determine a sequence of connected vertices $r[k] \in \mathcal{N}$ and control gains $K[k] \in \mathcal{K}, k=1, \cdots, l_{p}$, such that $r[1]$ satisfies the initial constraints, $r\left[l_{p}\right]$ satisfies the final constraints, and the path length $l_{p}$ is minimized.

Per the above algorithm, a graph search is utilized to determine the minimum number of equilibrium hops around a piece of debris. After the path has been determined as a sequence of the set-points and the corresponding control gains, the execution of the path proceeds by checking if the current state, $X(t)$ is in the safe positively invariant set corresponding to the next reference $r^{+}$and next control gain $K^{+}$in the sequence; if it is, then the controller switches to this reference and control gain:

$$
X(t) \in C\left(r^{+}, K^{+}, \rho^{*}\left(r^{+}, K^{+}, z\right)\right) \rightarrow r(t)=r^{+}, K(t)=K^{+} .
$$

\section{Simulation Results}

Simulations are now provided that illustrate our debris avoidance approach. We consider a nominal circular orbit of $850 \mathrm{~km}$ and discretize the HCW equations with a sampling period, $\Delta T$, of 30 seconds. We construct an approximately $2 \mathrm{~km}$ cubed virtual net. We let $R=2 \times 10^{7} I_{3}$ and $Q=$ $\operatorname{diag}\left(100,100,100,10^{7}, 10^{7}, 10^{7}\right)$.

An ellipsoidal set over-bounds a piece of debris, $O\left(z_{1}, Q_{1}\right)$, centered at $z_{1}=\left[\begin{array}{llll}0.3 & 0.4 & 0.5\end{array}\right]^{\mathrm{T}} \mathrm{km}$ and with $Q_{1}=100 I_{3}$. In this example, we use the fast distance computation technique of [1] based on bisections applied to (15) to determine the growth distance to the debris from each node in the net. The spacecraft's initial condition is $X(0)=X_{e}\left(r_{0}\right)$, where $r_{0}=\left[\begin{array}{lll}0.45 & 0 & 2.25\end{array}\right]^{\mathrm{T}}$. The target equilibrium node is $X_{e}(0)$. Finally, we impose a maximum thrust constraint of $10 \mathrm{~N}$ in each axis. Dijkstra's algorithm is used to find the shortest cost path from initial node to final node.

Figure 6 shows the path the spacecraft takes under closedloop control in order to avoid the debris. The spacecraft is able to complete the desired maneuver well within maximum thrust constraints while successfully avoiding the debris.

In Figure 7 we rerun the above simulation for a grid of initial conditions. The maneuvers shown in Figure 7 clearly demonstrate the initial conditions for which the maneuver path is perturbed from that which the spacecraft would have taken had there been no debris.

Finally, we add a second piece of debris, $O\left(z_{2}, Q_{2}\right)$, centered at $z_{2}=\left[\begin{array}{lll}-0.3 & 0.4 & 0.5\end{array}\right]^{\mathrm{T}}$ and with $Q_{2}=100 I_{3}$. In calculating the growth distance, we take the minimum distance to each of $O\left(z_{i}, Q_{i}\right), i=1,2$. Figure 8 shows the path the spacecraft takes under closed-loop control in order to avoid the debris.

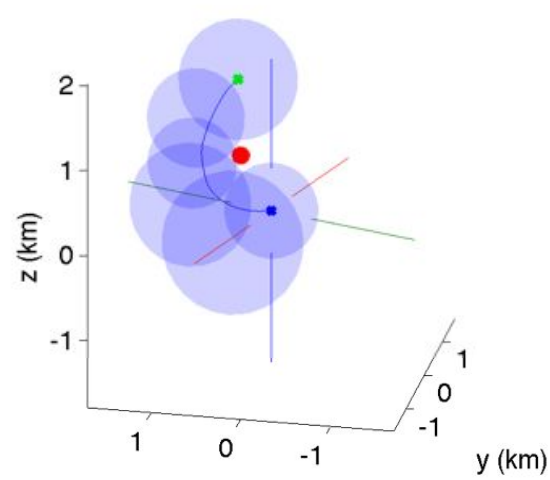

(a) Debris Avoidance Path.

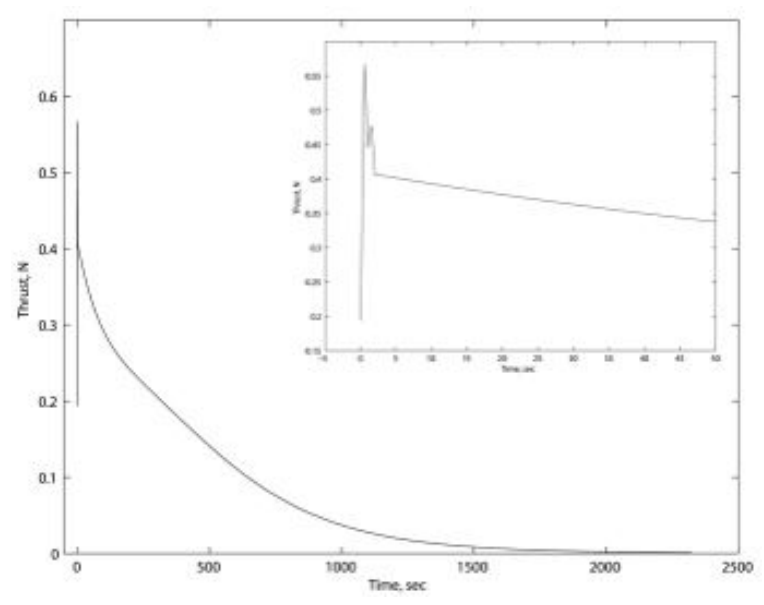

(b) Norm of Thrust Profile.

Fig. 6: Debris Avoidance Path. The green $\mathrm{x}$ marks the initial node. The blue $\mathrm{x}$ marks the final node. The red ellipsoid represents the debris. The blue line is the path the spacecraft takes in order to avoid the debris. The blue ellipsoids represent the invariant sets along the path.

\section{CONClusion}

This paper described a technique for spacecraft maneuver planning that uses positively-invariant sets in order to avoid collisions with debris, while adhering to specified thrust limits. The approach is based on hopping between neighborhoods of equilibria in a virtual net, and maintaining the spacecraft trajectory within a tube formed by safe positivelyinvariant sets. For the case where thrust limits can be specified as polyhedral norm bounds, we have shown that the thrust limit on the growth distance can be easily computed; it is, in fact, feasible to perform these computations onboard a spacecraft in order to account for thruster failure or degradation.

\section{REFERENCES}

[1] Baldwin, M., Weiss, A., Kolmanovsky, I., and Erwin, R.S.,"Spacecraft debris avoidance using constraint admissible positively invariant sets," Proceedings of AAS Space Flight Mechanics Meeting, Paper AAS 12250, Charleston, South Carolina, 2012. 


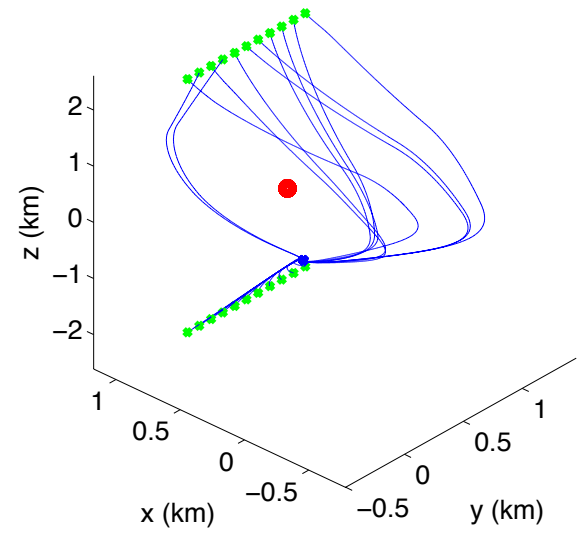

Fig. 7: Debris Avoidance Paths for many initial conditions. Each green $\mathrm{x}$ marks an intial condition. The blue $\mathrm{x}$ marks the final node. The red ellipsoid represents the debris. The blue lines are the paths that the spacecraft takes from each initial condition in order to avoid the debris. We do not show the invariant set ellipsoids for visual clarity.

[2] LaValle, S.M., Planning Algorithms, Cambridge University Press, 1996.

[3] Maia, M.H. and Galvao, R.K.H., "On the use of mixed-integer linear predictive control with avoidance constraints," International Journal of Robust and Nonlinear Control, Vol. 19, pp. 822-828.

[4] Ranieri, C"Path-constrained trajectory optimization for proximity operations," AIAA/AAS Astrodynamics Specialist Conference, Honolulu, HI, August 2008.

[5] Epenoy, R., "Fuel optimization for continuous-thrust orbital rendezvous with collision avoidance constraint," Journal of Guidance, Control, and Dynamics, Vol. 34, No. 2, March-April 2011.

[6] Sanchez-Ortiz, N., Bello-Mora, M., and Klinkrad, H., "Collision avoidance manoeuvres during spacecraft mission lifetime: Risk reduction and required $\Delta$ V," Advances in Space Research, Vol. 38, 2006.

[7] Martinson, N., Munoz, J., and Wiens, G., "A new method of guidance control for autonomous rendezvous in a cluttered space environment," Proceedings of AIAA Guidance, Navigation, and Control Conference, Hilton Head, SC, August 2007.

[8] Martinson, N.,"Obstacle avoidance guidance and control algorithms for spacecraft maneuvers," Proceedings of AIAA Guidance, Navigation, and Control Conference, Chicago, IL, August 2009.

[9] Mueller, J., Griesemer, P., and Thomas, S., "Avoidance maneuver planning incorporating station-keeping constraints and automatic relaxation," AIAA Infotech@Aerospace 2010, Atlanta, GA, April 2010.

[10] Ulybyshev, Y., "Trajectory optimization for spacecraft proximity operations with constraints," AIAA Guidance, Navigation, and Control Conference, Portland, OR, August 2011.

[11] Richards, A., Schouwenaars, T., How, J., and Feron, E., "Spacecraft trajectory planning with avoidance constraints using mixed-integer linear programming," AIAA Journal of Guidance, Control, and Dynamics, Vol. 25, No. 4, August 2002.

[12] Mueller, J.,"Onboard planning of collision avoidance maneuvers using robust optimization," AIAA Infotech@Aerospace 2009, Seattle, WA, April 2009.

[13] Kolmanovsky I.V., Gilbert E.G., "Theory and computation of disturbance invariant sets for discrete-time linear systems," Mathematical Problems in Engineering, Vol. 4(4), pp. 317-367, 1998.

[14] Wie, B., Spacecraft Dynamics and Control, 2nd Ed. Reston, VA: AIAA,2010.

[15] Kolmanovsky, I.V., and Gilbert, E., "Multimode regulators for systems with state and control constraints and disturbance inputs," Proceedings of Workshop Control Using Logic-Based Switching, pp. 104-117, published in an edited volume by Springer-Verlag, Editor: Morse A.S, Block Island, Rhode Island, Fall, 1997.

[16] Park, H., Di Cairano, S., and Kolmanovsky, I.V., "Linear Quadratic

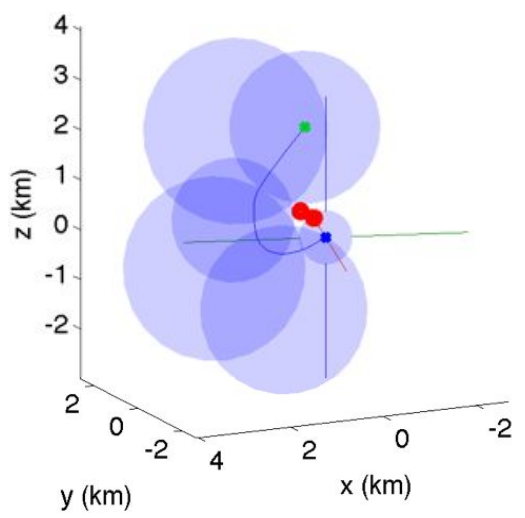

(a) Debris Avoidance Path.

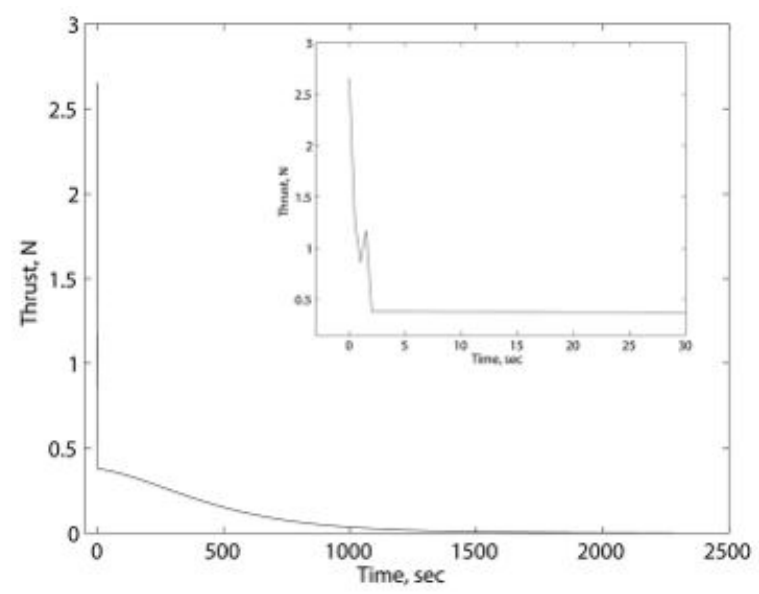

(b) Norm of Thrust Profile.

Fig. 8: Debris Avoidance Path for 2 pieces of debris. The green $\mathrm{x}$ marks the initial node. The blue $\mathrm{x}$ marks the final node. The red ellipsoid represents the debris. The blue line is the path the spacecraft takes in order to avoid the debris. The blue ellipsoids represent the invariant sets along the path.

Model Predictive Control approach to spacecraft rendezvous and docking," Proceedings of 21st AAS/AIAA Space Flight Mechanics Meeting, New Orleans, Louisiana, USA, Paper AAS-142, 2011.

[17] Ong C.J., Gilbert E.G., "Growth distances: New measures for object separation and penetration," IEEE Transactions on Robotics and Automation, Vol. 12(6), pp. 888-903, 1996. 